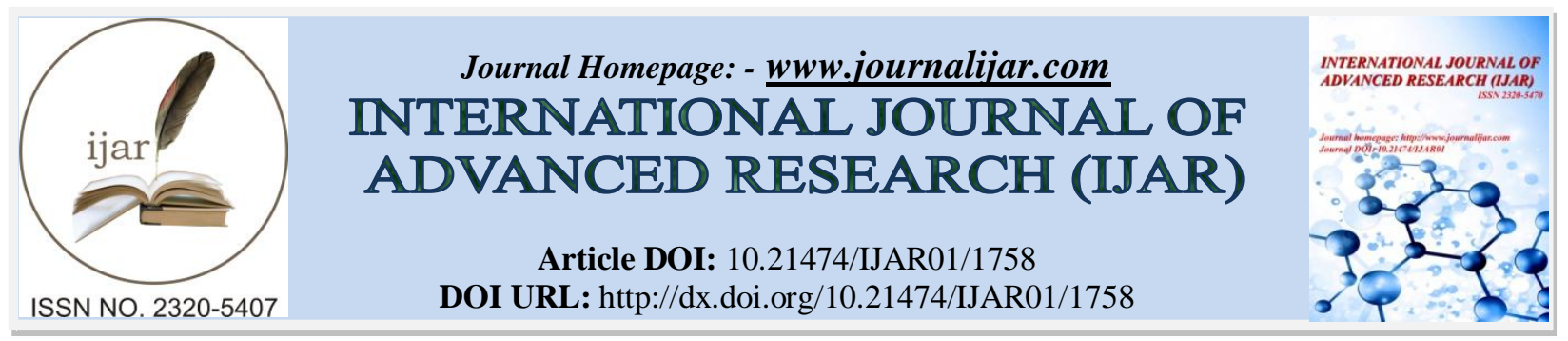

RESEARCH ARTICLE

\title{
CLINICAL APPLICATION OF THREE-COLUMN THEORY TO TIBIOFIBULAR SYNDESMOSIS INJURY AFTER ANKLE FRACTURES.
}

Sufang Huang, Chaoliang Wang and Xuesheng Sun.

Department of Orthopedics, People's Hospital of Laiwu City, Shandong Province, China.

\section{Manuscript Info}

Manuscript History

Received: 12 August 2016

Final Accepted: 22 September 2016

Published: October 2016

Key words:-

Ankle fractures, tibiofibular syndesmosis injury, internal fixation, functional assessment

\section{Abstract}

Objective:- To explore the clinical effects of internal fixation on ankle fractures combined with tibiofibular syndesmosis injury under the guidance of three-column theory.

Methods:- Clinical data of 54 patients with ankle fractures combined with tibiofibular syndesmosis injury, who were treated from June 2008 to May 2014, were retrospectively analyzed in this study. According to the characteristics of pathological anatomy and mechanisms of injury, ankle joint was divided into three columns. Above three columns were involved when ankle fractures combined with tibiofibular syndesmosis separation appeared. There were lateral column fracture in 54 sites, ligament injury of middle column in 26 sites, avulsion fracture of the middle column in 28 sites, ligament injury of inside column in 31 sites, and inside column fracture in 13 sites. At least two columns should be fixed after the three columns were injured. Visual analogue scale pain scores were recorded at 6 months after surgery. Imaging results were evaluated using modified Baird and Jackson evaluation criteria after fracture reduction and fixation. The function of ankle joint was assessed utilizing American Orthopaedic Foot and Ankle Society ankle-hindfoot score system.

Results:- A total of 54 patients were followed up for 6-48 months (averagely 21 months). Healing time was 11-15 weeks (averagely 13.6 weeks). Visual analogue scale pain scores were between 0 and 6 (averagely 1.4). American Orthopaedic Foot and Ankle Society anklehindfoot score was 82-100, averagely 96. Radiological evaluation of therapeutic effects showed 52 excellent cases and 2 good cases with an excellent and good rate of $100 \%$.

Conclusion:- Three-column classification for ankle fractures is a simple and comprehensive theory. Fixation of two columns can stabilize the tibiofibular syndesmosis after three columns were injured.

Copy Right, IJAR, 2016,. All rights reserved.

\section{Introduction:-}

Ankle can be easily injured during sports [1]. Ankle fractures frequently combine with ligament injuries surrounding the ankle joint. Approximately 10\% patients with ankle fractures combined with tibiofibular syndesmosis injury [2]. Complete tibiofibular syndesmosis is important to maintain a stable structure of the ankle joint. If talus moves $1 \mathrm{~mm}$ 
outward and the contact surface of tibial Astragaloside joint reduces 42\%, the probability of occurrence of traumatic arthritis will increase [3]. Improper treatment for tibiofibular syndesmosis injury will cause long-term ankle pain, even disability. Ankle fractures combined with tibiofibular syndesmosis separation is challenging in clinical treatment. A previous study confirmed that fixation was still necessary during unstable tibiofibular syndesmosis after fixation for ankle fractures [4]. However, there is no simple effective method to choose a surgical approach or fixation method. Ankle fracture types commonly used in clinic are AO-Danis-weber classification and LangerHanse classification. Above types are too simple or too cumbersome, difficult to remember, do not cover all types of fractures, and provide limited assistance to the determination of the surgical program for ankle fractures combined with tibiofibular syndesmosis injury. We proposed the three-column theory to guide ankle fractures combined with tibiofibular syndesmosis injury. Among the three columns, the stabilization of two columns can achieve the fixation of the tibiofibular syndesmosis. The present study retrospectively analyzed complete information on 54 patients with ankle fractures, who were treated in our hospital from June 2010 to May 2014, and sought to observe characteristics of ankle fractures combined with tibiofibular syndesmosis injury and mechanisms of injury and to explore the clinical effects of internal fixation on above injuries under the guidance of three-column theory.

\section{Data and methods:- General data:-}

A total of 54 ankle fracture patients with complete follow-up data, who were treated from June 2008 to May 2014 , were retrospectively analyzed. There were 43 males and 11 females, at the age of 21-58 years old, averagely 36.7 years old. The causes of injuries contain high fall injuries in 23 cases, traffic injuries in 16 cases, sports injuries in 11 cases, and other injuries in 4 cases. These patients affected closed fractures. According to Langer-Hanse classification, there were supination-external rotation in 20 cases, supination and adduction in 14 cases, pronation and abduction in 8 cases, and pronation-external rotation in 4 cases. Patients with acute open injury were subjected to debridement, reduction and fixation during emergency surgery. In this study, tibiofibular syndesmosis separation is identified by following signs: obvious local pain, widened inferior tibiofibular gap at ankle points shown by Xray, which was $2 \mathrm{~mm}$ wider than normal control. CT images in the axial plane revealed that the difference of anterior and posterior tibiofibular gaps was more than $2.0 \mathrm{~mm}$ [5]. During the surgery, patients were diagnosed as tibiofibular syndesmosis separation by retractor test.

\section{Principles of management:-}

After hospitalization, the patient should raise the affected limb and receive cold compress. Closed injury patients should make an operation within 6-8 hours after injury. For local swelling patients, a brace should be used to fix the affected limb until detumescence (7-14 days after injury). Surgery could be conducted when soft tissue conditions were improved and skin wrinkles appeared. X-ray and CT three-dimensional reconstruction were performed.

\section{Definition of three columns of the ankle joint:-}

The three columns are composed of lateral column, inside column and middle column, which is classified according to the structure of ankle joint. The lateral column consists of lateral ligament complex (lateral A), distal fibula (lateral B), fibular tibiofibular syndesmosis level (lateral C) and proximal fibula (lateral D) from far to near. The inside column consists of deltoid ligament (inside A) and inside malleolus (inside B) from far to near. The middle column consists of tibiofibular syndesmosis ligament (anterior tibiofibular ligament, posterior tibiofibular ligament, transverse tibiofibular ligament and interosseous ligament) and interosseous membrane (middle A) and anterior tibial attachment site of tibiofibular syndesmosis (middle B, anterior tibia, Tillaux fracture) or posterior tibial attachment site (middle B, posterior tibia), anterior fibular attachment site of tibiofibular syndesmosis (middle B, anterior fibula, Wagstaffe fracture) [6] or posterior fibular attachment site (middle B, posterior fibula) (Figure 1). Shift direction of distal fracture after ankle fracture contains outward shift and inward shift. According to sites of fibula fracture, outward displacement is composed of lateral $\mathrm{C}+$ middle $\mathrm{A}+$ inside $\mathrm{B}$ (Figure 2), lateral $\mathrm{C}+$ middle B + inside B (Figure 3), lateral D + middle A + inside A (Figure 4), lateral D + middle A + inside B (Figure 5), lateral D + middle B + inside A (Figure 6), and lateral D + middle B + inside B (Figure 7). 


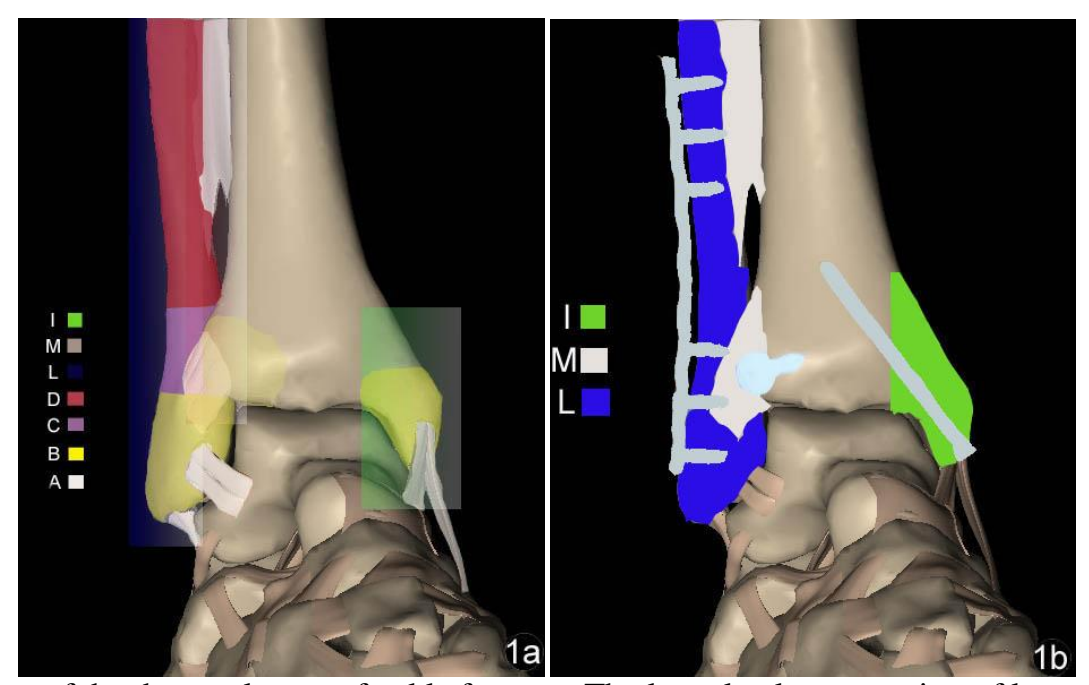

Figure 1a. Definition of the three columns of ankle fracture. The lateral column consists of lateral ligament complex (lateral A), distal fibula (lateral B), fibular tibiofibular syndesmosis level (lateral C) and proximal fibula (lateral D) from far to near. The inside column consists of deltoid ligament (inside A) and inside malleolus (inside B) from far to near. The middle column consists of tibiofibular syndesmosis ligament (anterior tibiofibular ligament, posterior tibiofibular ligament, transverse tibiofibular ligament and interosseous ligament) and interosseous membrane (middle A) and anterior tibial or fibular attachment site of tibiofibular syndesmosis (middle B). Figure 1b. Each column fracture was fixed using screw or plate. At least two columns should be fixed after ankle fractures combined with tibiofibular syndesmosis separation.

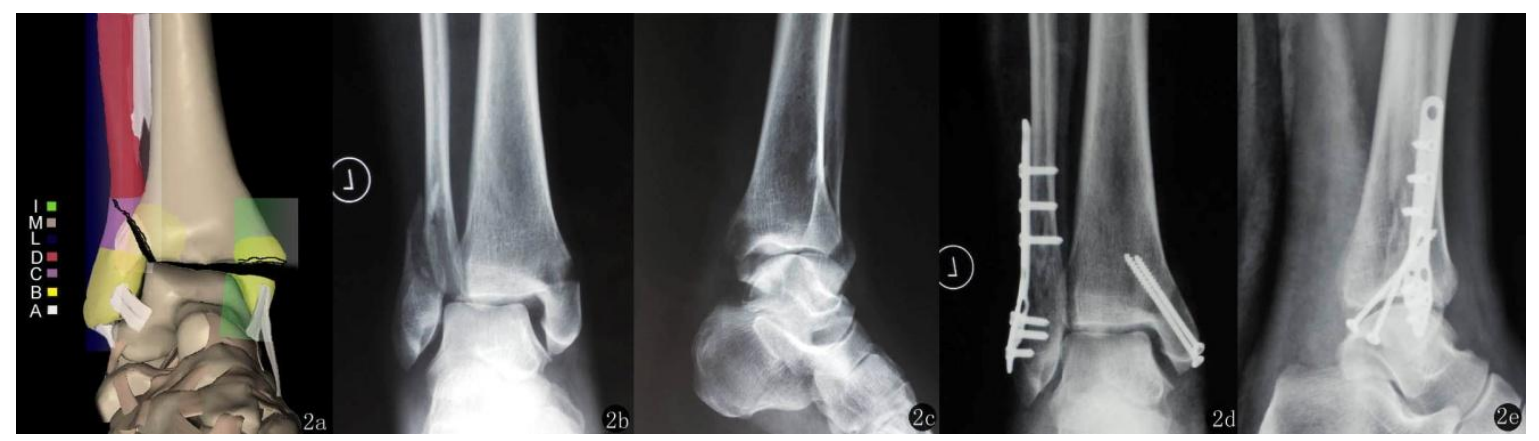

Figure 2. Fracture cases at lateral column $\mathrm{C}+$ middle column $\mathrm{A}+$ inside column B. Fracture diagram (A); using fixation through lateral ankle approach + inside approach, preoperative anteroposterior (B) and lateral (C) radiographs; postoperative anteroposterior (D) and lateral (E) radiographs.

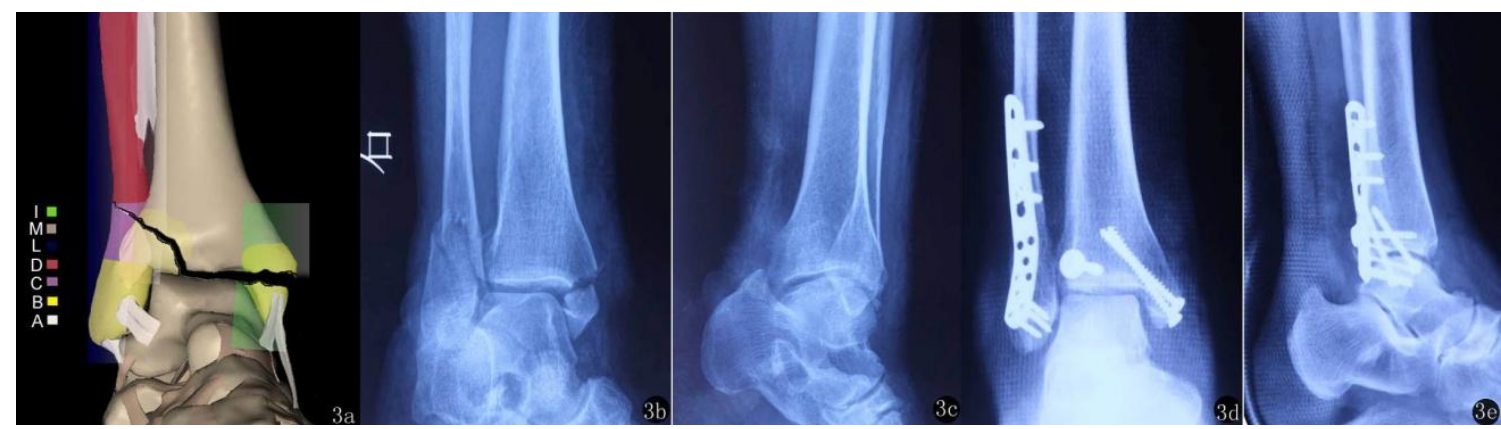

Figure 3:- Fracture cases at lateral column C + middle column B + inside column B. Fracture diagram (A); using fixation through posterolateral ankle approach + inside approach, preoperative anteroposterior (B) and lateral (C) radiographs; postoperative anteroposterior (D) and lateral (E) radiographs. 


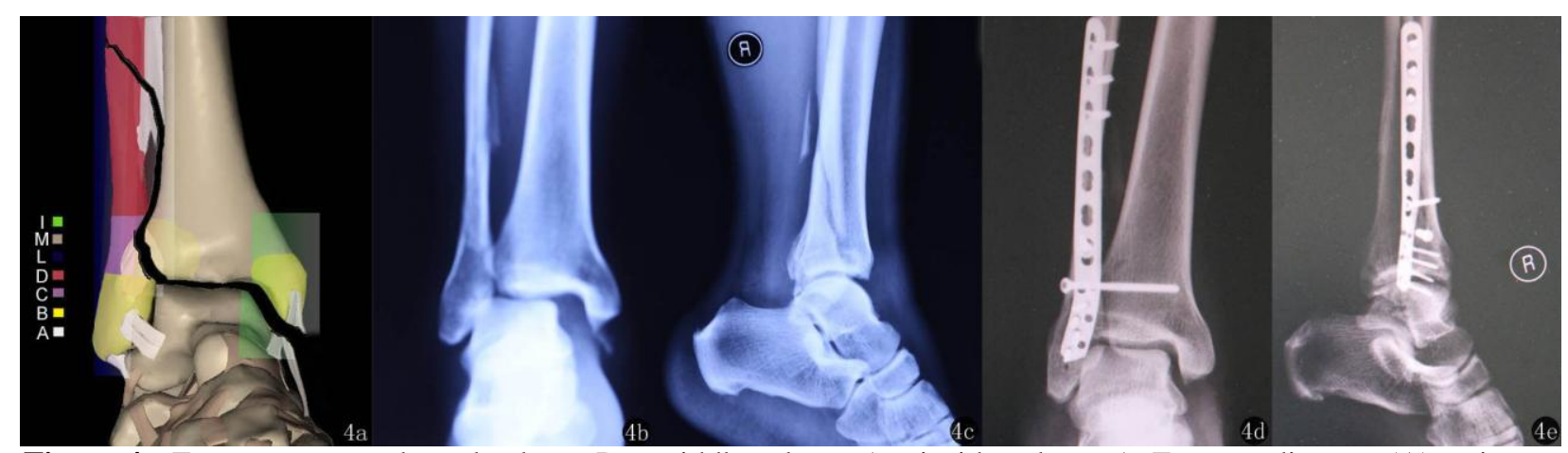

Figure 4:- Fracture cases at lateral column D + middle column A + inside column A. Fracture diagram (A); using fixation through posterolateral ankle approach, preoperative anteroposterior (B) and lateral (C) radiographs; postoperative anteroposterior (D) and lateral (E) radiographs.

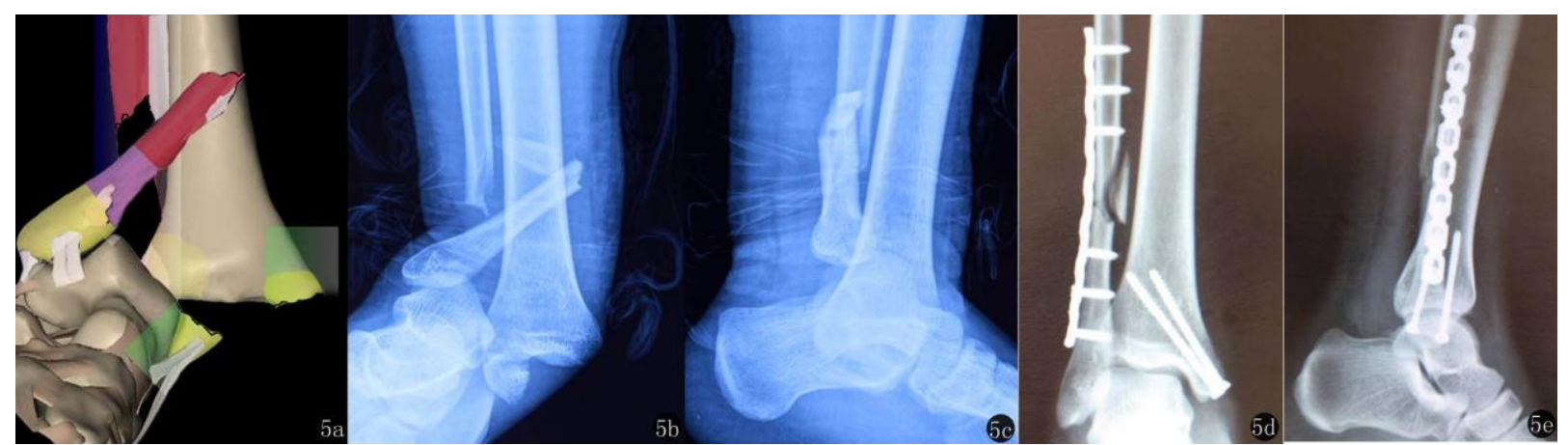

Figure 5:- Fracture cases at lateral column D + middle column A + inside column B. Fracture diagram (A); using fixation through lateral ankle approach + inside approach, preoperative anteroposterior (B) and lateral (C) radiographs; postoperative anteroposterior (D) and lateral (E) radiographs.
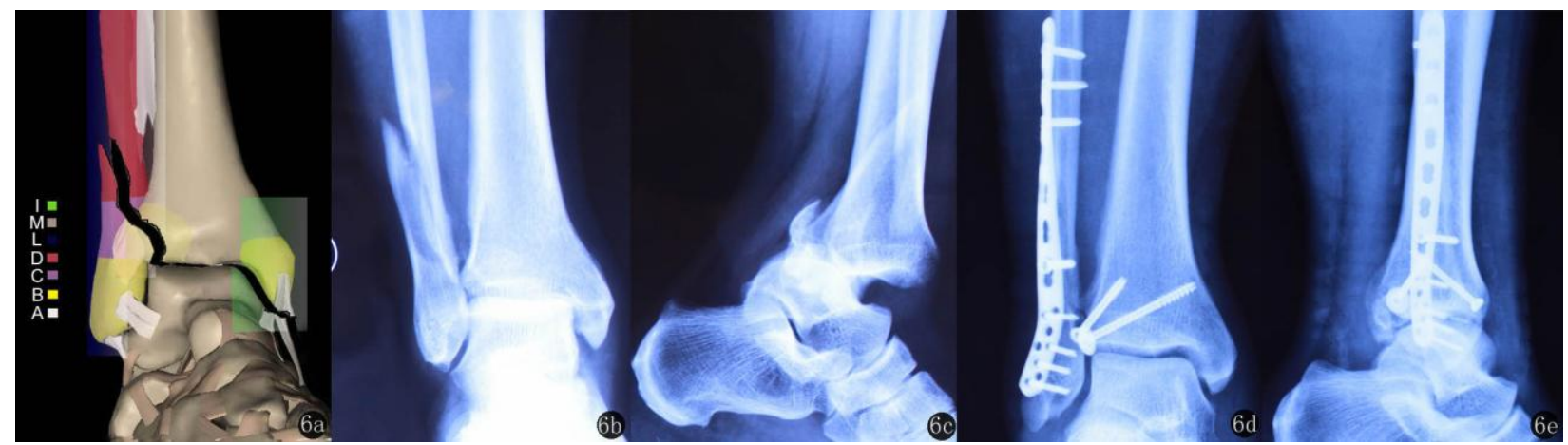

Figure 6:- Fracture cases at lateral column D + middle column B + inside column A. Fracture diagram (A); using fixation through posterolateral ankle approach + anterolateral auxiliary incision, preoperative anteroposterior (B) and lateral (C) radiographs; postoperative anteroposterior (D) and lateral (E) radiographs. 


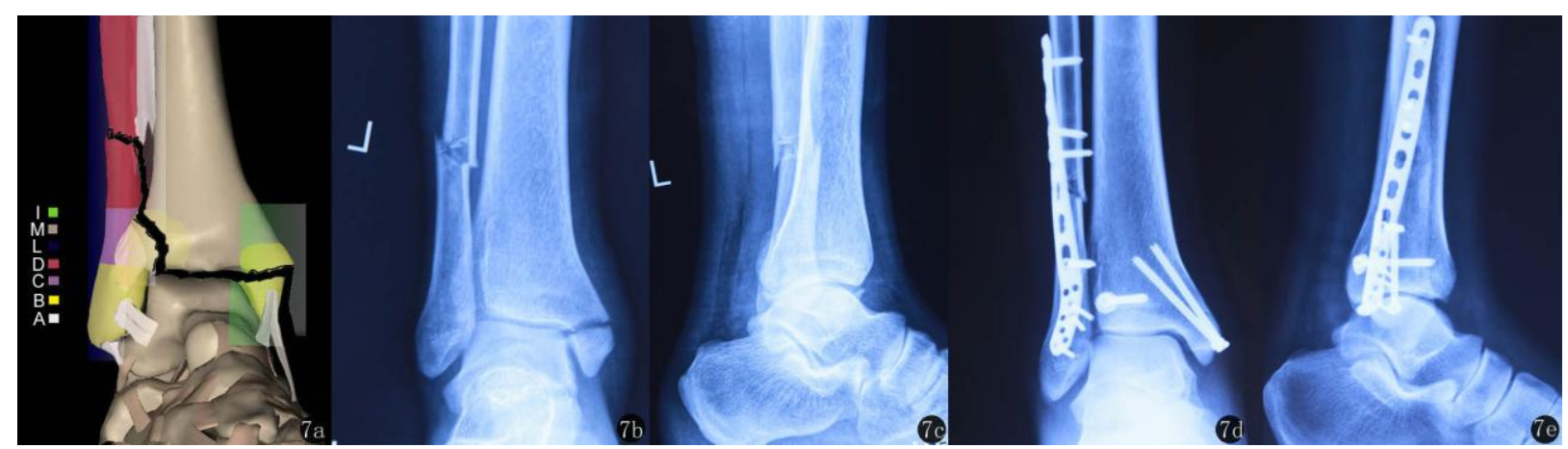

Figure 7:- Fracture cases at lateral column D + middle column B + inside column B. Fracture diagram (A); using fixation through posterolateral ankle approach + inside approach, preoperative anteroposterior (B) and lateral (C) radiographs; postoperative anteroposterior (D) and lateral (E) radiographs.

Table 1:- Distribution of patients factors.

\begin{tabular}{|c|c|c|c|c|}
\hline \multirow{2}{*}{\multicolumn{2}{|c|}{ Patients' characteristic }} & \multicolumn{3}{|c|}{ Three column classification } \\
\hline & & \multirow{2}{*}{$\begin{array}{l}\mathrm{IB}+\mathrm{MA}+\mathrm{LC} \\
26\end{array}$} & \multirow{2}{*}{$\begin{array}{l}\mathrm{IB}+\mathrm{MAB}+\mathrm{LD} \\
22\end{array}$} & \multirow{2}{*}{$\begin{array}{l}\text { IB+MBB+LD } \\
6\end{array}$} \\
\hline Fractures & 54 & & & \\
\hline Gender, No. males & 43 & 19 & 15 & 9 \\
\hline Age, years (range) & $37(21-57)$ & $36(22-56)$ & $42(24-58)$ & $35(21-53)$ \\
\hline Right side & 32 & 14 & 11 & 7 \\
\hline Result of fall & 23 & 11 & 7 & 5 \\
\hline $\begin{array}{l}\text { Result of motor vehicle } \\
\text { accident }\end{array}$ & 16 & 8 & 5 & 3 \\
\hline Result of sport injury & 11 & 6 & 3 & \multirow[t]{2}{*}{2} \\
\hline \multicolumn{4}{|l|}{ Operative characteristics } & \\
\hline $\begin{array}{l}\text { Time from injury to } \\
\text { surgery, days }\end{array}$ & $9.53 \pm 3.12$ & $9.19 \pm 4.16$ & $8.11 \pm 2.14$ & $10.06 \pm 5.18$ \\
\hline Hospital stay, days & $17.32 \pm 2.18$ & $16.14 \pm 4.37$ & $17.05 \pm 3.19$ & $17.54 \pm 3.14$ \\
\hline surgical approach & & $\mathrm{I}+\mathrm{L}$ & $\mathrm{I}+\mathrm{AL}$ & $\mathrm{I}+\mathrm{BL}$ \\
\hline $\begin{array}{l}\text { column of internal } \\
\text { fixation }\end{array}$ & & $\mathrm{I}+\mathrm{L}$ & $\mathrm{I}+\mathrm{MA}+\mathrm{L}$ & $\mathrm{I}+\mathrm{MB}+\mathrm{L}$ \\
\hline Functional results & & Excellent $(2$, & excellent & excellent \\
\hline
\end{tabular}

I, inside column; M, middle column; L, lateral column; B, before column; A, after column

\section{Surgical approaches and steps:-}

A surgical approach was selected according to different injured columns. Lateral approach to the fibula was selected for lateral column fracture alone. Standard approach to the inside malleolus was selected for inside column fracture. Posterolateral approach was selected for fractures of lateral column combined with posterior inferior tibiofibular ligament tibial avulsion. Anterolateral approach was selected for fractures of lateral column combined with anterior inferior tibiofibular ligament tibial avulsion. An incision was used as possible to solve the problem. Procedures are as follows: (1) fractures of inside malleolus were fixed using double lag screws or wire tension band. Fractures of posterior or anterior ankle were fixed using lag screw or hollow screw. Three layers of cortex were fixed with one or two cortical bone screws in the form of a lag screw from the top of the tibiofibular syndesmosis and paralleled with the ankle mortise in patients with tibiofibular syndesmosis separation. (2) At least two columns were fixed in patients with ankle fractures combined with tibiofibular syndesmosis separation. Fractures should be fixed first. The tibiofibular syndesmosis could be fixed with a screw for ligament rupture-induced tibiofibular syndesmosis separation.

\section{Postoperative treatment and efficacy evaluation:-}

After surgery, short leg plaster was applied for fixation for 4 weeks. Ankle joint moved positively or passively to restore the range of motion of the ankle joint. At 6 weeks after surgery, patients could bear weight partially. Radiographs revealed that patients could accept full weight bearing after fracture healing. During follow-up, visual analogue scale was employed to assess patient's pain. American Orthopaedic Foot and Ankle Society, ankle- 
hindfoot score system, was utilized to evaluate the function of ankle joint [7, 8]. Curative effects were evaluated: excellent 91-100 points, good 81-90 points, average 60-80 points, and poor $<60$ points. Score was measured in all patients at 6 months after surgery according to modified Baird and Jackson subjectivity and objectivity and X-ray [9].

At the final follow-up, all patients were reevaluated using VAS, AOFAS, and modified Baird and Jackson subjectivity and objectivity and X-ray.

\section{Results:-}

A total of 54 cases were followed up for 6-48 months (averagely 21 months). The wound healed well, no infection or local adverse reaction was visible. Healing time was 11-15 weeks (averagely 13.6 weeks). Visual analogue scale pain scores were between 0 and 6 (averagely 1.4). American Orthopaedic Foot and Ankle Society ankle-hindfoot score was 82-100, averagely 96. Radiological evaluation of therapeutic effects showed 52 excellent cases and 2 good cases with an excellent and good rate of $100 \%$. In accordance with the three-column theory, ankle injury combined with tibiofibular syndesmosis separation belonged to three-column injuries, and the fixation of two columns could stabilize the tibiofibular syndesmosis.

\section{Discussion:-}

\section{Ankle fracture types:-}

Ankle fracture types commonly used in clinic are Langer-Hanse classification [10, 11] and AO-Danis-weber classification [12]. In accordance with mechanisms of ankle injuries, the position of the foot when damage occurs, and the direction of rotation in the ankle mortise, Langer-Hanse classification includes five types: supinationexternal rotation, supination-adduction, pronation-abduction, rotation-external rotation and vertical compression. Langer-Hanse classification only describes ankle injury mechanism, but the mechanisms of ankle fractures are diverse. Langer-Hanse classification system is better, but still cannot contain all kinds of ankle injuries [13]. The types 1 and 2 of pronation-external rotation and pronation-abduction cannot be distinguished in Langer-Hanse classification [14]. In this study, 12.9\% (8/54) ankle injuries could not be classified according to Langer-Hanse classification. We could not tell the type 1 of pronation-external rotation from type 1 of pronation-abduction in four patients, and could not distinguish type 2 of pronation-external rotation from type 2 of pronation-abduction in two patients with inside malleolus fractures combined with anterior tibiofibular ligament injury. Lauge-Hansen classification appears early based on the mechanism of violence injury and its core is to identify ankle fracture mechanism in order to establish closed reduction program. The effects of internal fixation that was widely used in recent years are limited in guiding surgery program. Internal fixation does not have clear guidance in the choice of incision and implants.

Ankle AO classification is mainly based on the positional relationship between fibula fracture level and tibiofibular syndesmosis ligament, and carefully describes ankle injury [15]. Ankle fractures are classified into three types and corresponding subtypes: type A: fibula fracture below tibiofibular syndesmosis plane; A1: fibula fracture alone, A2: combined with inside malleolus injury, A3: combined with posterior inside fracture. Type B: fibula fracture on tibiofibular syndesmosis plane; B1: fibula fracture alone, B2: combined with inside injury, B3: combined with inside injury and posterolateral tibia fracture. Type C: fibula fracture above tibiofibular syndesmosis plane; C1: fibular shaft fractures alone, C2: compound fracture of the fibular shaft, C3: proximal fibula fracture. The type A of AO classification refers to supination adductor injury. The type $\mathrm{B}$ of $\mathrm{AO}$ classification refers to supination-external rotation injury. The type $\mathrm{C}$ of $\mathrm{AO}$ classification refers to pronation-abduction and pronation-eversion injury [16]. AO classification based primarily on the fracture site is a detailed and comprehensive classification, and facilitates the acquisition and management of cases, but ignores the ligament surrounding the ankle and the structure of tibiofibular syndesmosis. AO classification is not associated with anatomical characteristics and mechanisms of fractures, and cannot guide to choose treatment programs.

Ankle injuries are mostly caused by low-energy rotation injury, and different from Pilon fracture induced by the axial load of distal tibial articular surface [17]. In the present study, low-energy injury induced by falls and sprains accounted for $77.8 \%$ (42/54). Coronal, sagittal and horizontal CT scan revealed fracture line mainly distributed along front and rear direction of the fibula, inside malleolus and lateral malleolus, as well as tibial attachment point of inferior tibiofibular ligament ending point. To further understand the mechanisms of ankle fractures and to guide clinical surgery, the three-column theory of ankle joint was used to guide the surgery in 54 patients with ankle 
fractures. We found that nearly all cases can be distinguished by this three-column theory, which consists of all types of ankle injuries, such as unusual Maisonneuve fracture, Tillaux fractures, and Wagstaffe fracture. Thus, missed diagnosis can be avoided. The core of the three-column theory is to consider the distal bone and ligament structure of ankle fractures as a complex. According to the direction of complex shift inward or outward, the type of fractures is analyzed and surgery program is determined. The effects of ligament and tibiofibular syndesmosis structure in ankle injuries should be paid attention. Follow-up results showed that clinical effects were good in 54 patients with ankle injuries.

\section{Surgical approaches and fixation:-}

One advantage of the three-column theory is to directly select the corresponding surgical approach according to fractures of different columns. (1) Lateral column fractures: fibula anatomical plate, reconstruction plate or 1/3 tubular plate is used through fibula lateral approach. (2) Fibula anterolateral or posterolateral approach for fractures involving the column: anatomical distal fibula plate is utilized through posterolateral approach by entering the posterior gap of peroneus longus and brevis muscles. Compression screw or hollow screw is applied for middledolumn avulsion fractures. Using anterolateral approach, a curved incision is made along anterolateral fibula. Extensor retinaculum is cut open. The extensor digitorum longus tendon, deep peroneal nerve and anterior tibial artery are retracted insidely so as to expose fractures of the lateral column. Fibula is fixed with reconstruction plate or 1/3 tubular plate. For the middle-column fracture, lag screw or bone plate is utilized according to the size of fracture blocks. (3) Fractures of inside column: using anteroinside or inside tibial approach, a curved incision is made in the inside malleolus. Fractures were fixed with two malleolus screws or wire tension band. It is easy to expose fractures through inside approach, but it is important to protect saphenous nerve and saphenous vein.

Guidance of the three-column theory for ankle fractures combined with tibiofibular syndesmosis injury:-

Tibiofibular syndesmosis injury is commonly associated with ankle fractures. Violent abduction and external rotation are main reasons for tibiofibular syndesmosis injury. Tibiofibular syndesmosis separation will increase the width of ankle mortise, and increase the range of motion of talus in the ankle mortise, resulting in unstable ankle joint. Early correct diagnosis and treatment of tibiofibular syndesmosis injury is the key for the recovery of ankle function. Presently, the diagnosis and treatment of tibiofibular syndesmosis injury are controversial. More and more basic and clinical studies suggested that not all separated tibiofibular syndesmosis should be fixed [18]. Unstable ankle mortise after tibiofibular syndesmosis separation and subsequent traumatic arthritis are worrisome. At present, screws are commonly used to fix tibiofibular syndesmosis separation. Following tibiofibular syndesmosis separation, the function of fibula to restrict talus relocation weakens. Talus relocation during weight-bearing walking will reduce the contact area of tibiotalar joint and increase local stress of the joint. Nevertheless, the stability of ankle mortise is maintained by various factors, involving lateral malleolus, lateral ligament complex, inside malleolus, inside deltoid ligament, tibiofibular syndesmosis, interosseous membrane, even calf muscles. Tibiofibular syndesmosis separation is just the manifestations of inferior tibiofibular ligament tear or ligament avulsion fractures. Structural integrity of the inside ankle talus can ensure that talus does not shift in the ankle mortise. Therefore, as long as anatomical reduction and stable fixation of the inside malleolus and lateral malleolus are conducted, the tibiofibular syndesmosis can reset itself. Even if there is a potential separation, the fixation is not needed if tibiofibular gap is less than $4 \mathrm{~mm}$. In this study, ankle injuries combined with tibiofibular syndesmosis separation belongs to three-column injuries. Moreover, the fixation of two columns can stabilize the tibiofibular syndesmosis. The fixation is required for the following situations: (1) inferior tibiofibular ligament injury does not need to be repaired, but open reduction and screw fixation are needed for posterior inferior tibiofibular ligament avulsion fractures at the tibial attachment site or anterior inferior tibiofibular ligament avulsion fractures at the tibial attachment site (Tillanx fracture), as well as rare anterior inferior tibiofibular ligament avulsion fractures at the fibular attachment site (Wagstaffe fracture). (2) Fractures of anterior tubercle of inside malleolus, simultaneously deep deltoid ligament rupture (from intertubercular sulcus and posterior tubercle, mainly posterior tibiotalar ligament and middle tibiotalar ligament) exist with triangular ligament rupture. (3) In three-column classification, lateral column $\mathrm{D}+$ middle column $\mathrm{A}+$ inside column $\mathrm{A}$, i.e., Maisonneuve fracture. Fixation with a screw in the tibiofibular syndesmosis can achieve excellent effects. (4) In three-column classification, lateral column A + middle column A + inside column A, i.e., tibiofibular syndesmosis separation without fractures. (5) Gap exists in old separated tibiofibular syndesmosis and avulsed bone surface or scar is filled in the gap, so it is necessary to scavenge the gap before fixation. (6) In three-column classification, tibiofibular syndesmosis belongs to ligament injury, i.e., middle column A. Tibiofibular syndesmosis separation still exists after inside and lateral columns are fixed, which may be caused by avulsion fracture blocks in the inferior tibiofibular gap. Thus, it is necessary to clear the gap before fixation. 
The treatment of ankle fractures combined with tibiofibular syndesmosis separation is the difficulty and focus in department of orthopedics and traumatology. Its fracture type is complicated. Using the three-column theory of fractures, we can simply find the fracture site. To pay close attention to the effects of ankle ligament and tibiofibular syndesmosis on fractures can effectively guide the surgery of ankle fractures. In fact, fractures will not follow a subjective mode. Any theory needs to be improved continuously in order to facilitate clinical treatment. The number of cases in this study is limited. Although encouraging results are obtained in initial stage, it still lacks large longterm follow-up data. The three-column theory of ankle fractures still needs further investigations and improvement.

\section{References:-}

1. Brosky T, Nyland J, Nitz A, Caborn DN. The ankle ligaments: consideration of syndesmotic injury and implications for rehabilitation. J Orthop Sports Phys Ther 1995; 21: 197-205.

2. Thornes B, Shannon F, Guiney AM, Hession P, Masterson E. Suture-button syndesmosis fixation: accelerated rehabilitation and improved outcomes. Clin Orthop Relat Res 2005; 431:207-212.

3. Ramsey PL, Hamilton W. Changes in tibiotalar area of contact caused by lateral talar shift. J Bone Joint Surg Am 1976; 58: 356-357.

4. Park JW, Kim SK, Hong JS, Park JH. Anterior tibiofibular ligament avulsion fracture in weber type B lateralmalleolar fracture. J Trauma 2002; 52: 655-659.

5. Gardner MJ, Demetrakopoulos D, Briggs SM, Helfet DL, Lorich DG. Malreduction of the tibiofibular syndesmosis in ankle fraetures. Foot Ankle Int 2006; 27: 788-792.

6. Wagstaffe WW. An unusual form of fracture of the fibula. Thomas hosp Rep 1875; 6: 43.

7. Mann G, Nyska M, Hetsroni I, Karlsson J. Scoring systems for evaluating ankle function. Foot Ankle Clin 2006; 11: 509-519.

8. Lee MS, Maker JM. Revision of failed flatfoot surgery. Clin Podiatr Med Surg 2009; 26: 47-58.

9. Baird RA, Jackson ST. Fracture of the distal part of the fibula with associated disruption of the deltoid ligament treatment without repair of the deltoid ligament. J Bone Joint Surg Am 1987; 69: 1346-1352.

10. Lauge-hansen N. Fractures of the ankle. III. Genetic roentgenologic diagnosis of fractures of the ankle. Am J Roentgenol Radium Ther Nucl Med 1954; 71: 456-471.

11. Lauge-hansen N. Fractures of the ankle IV. Clinical use of genetic roentgen diagnosis and genetic reduction. Arch Surg 1952; 64: 488-500.

12. Muller ME, Allgower M, Schneider R, et al. Manual of internal fixation. Technique Recommended by the AO Group. New York: Springer; 1979.

13. Alexandropoulos C, Tsourvakas S, Papachristos J, Tselios A, Soukouli P. Ankle fracture classification: an evaluation of three classification systems: Lauge-Hansen, AO and Broos-Bisschop. Acta Orthop Belg 2010; 76: 521-525.

14. Lindsjö U. Classification of ankle fractures: the Lauge-Hansen or AO system? Clin Orthop Relat Res 1985; 199: 12-16.

15. Court-Brown CM, McBirnie J, Wilson G. Adult ankle fractures: an increasing problem? Acta Orthop Scand 1998; 69: 43-47.

16. Clare MP. A rational approach to ankle fractures. Foot Ankle Clin 2008; 13: 593-610.

17. Sakaki MH, Matsumura BA, Dotta Tde A, Pontin PA, Dos Santos AL, Fernandes TD. Epidemiologic study of ankle fractures in a tertiary hospital. Acta Ortop Bras 2014; 22: 90-93.

18. Kennedy JG, Soffe KE, Dalla Vedova P, Stephens MM, O'Brien T, Walsh MG, McManus F. Evaluation of the syndesmotic screw in low Weber C ankle frankle fracture. J Orthop Trauma 2000; 14: 359-366. 